\title{
Critical Response of a Quantum van der Pol Oscillator
}

\author{
Shovan Dutta $\odot^{*}$ and Nigel R. Cooper \\ T.C.M. Group, Cavendish Laboratory, University of Cambridge, JJ Thomson Avenue, Cambridge CB3 OHE, United Kingdom
}

(Received 7 August 2019; published 20 December 2019)

\begin{abstract}
Classical dynamical systems close to a critical point are known to act as efficient sensors due to a strongly nonlinear response. We explore such systems in the quantum regime by modeling a quantum version of a driven van der Pol oscillator. We find the classical response survives down to one excitation quantum. At very weak drives, genuine quantum features arise, including diverging and negative susceptibilities. Further, the linear response is greatly enhanced by using a strong incoherent pump. These results are largely generic and can be probed in current experimental platforms suited for quantum sensing.
\end{abstract}

DOI: 10.1103/PhysRevLett.123.250401

A key insight from the theory of phase transitions is that systems close to a critical point are highly sensitive to perturbations [1]. This is evident, for example, in a diverging compressibility near a liquid-gas phase transition. Such divergences in susceptibility also occur in classical dynamical systems close to a bifurcation [2]. A particularly important prototype is an oscillator with nonlinear damping, which can transition from a dormant to a limit-cycle phase across a Hopf bifurcation [3]. Generically, the response of such a van der Pol (vdP) oscillator [4] to a resonant drive $\Omega$ grows as $\Omega^{1 / 3}$ at the critical point [5]. This nonlinearity is what enables the ear and other biological sensors to detect very weak stimuli and to maximally process environmental inputs [5-9].

In this Letter, we ask whether this increased sensitivity persists into the quantum regime. This could have important applications in the growing frontier of quantum sensing $[10,11]$. Besides, understanding the dynamics of a quantum vdP oscillator is also of fundamental interest, as it represents an easy-to-implement nonequilibrium setting where coherent drive competes with incoherent dissipation. Such open quantum systems are at the forefront of modern physics $[12,13]$, led by advances in synthetic experimental platforms with unprecedented control and tunability [14-18].

At the semiclassical level, the vdP oscillator is realized in the physics of lasers [19,20]. Recent theoretical studies have also examined a quantum vdP oscillator, largely in the context of synchronization [21-27]. However, its critical properties and response are as yet unexplored. Here, we fully characterize the response of a driven quantum vdP oscillator by a standard master equation, finding surprising features which can be probed in experiments.

We find the classical response persists all the way down to one excitation quantum. At weaker drives, the quantum oscillator exhibits divergent as well as negative susceptibilities. Furthermore, in the limit-cycle phase, the linear response is limited only by two-body loss, providing a strong sensitivity enhancement over a passive system.
Several of these features originate from a competition of energy scales and should generalize to other systems. After briefly reviewing the iconic classical vdP oscillator, we present results for the quantum response, concluding with a discussion of experimental realizations.

The classical vdP model describes a harmonic oscillator with nonlinear damping, $\ddot{x}=-\omega^{2} x+\left(2 \gamma_{1}-8 \gamma_{2} x^{2}\right) \dot{x}$. Here, $\omega$ is the natural frequency, $\gamma_{1}$ is a negative damping which may arise from an energy source, and $\gamma_{2}>0$ is the leading nonlinear term which damps the system at large $x$. For $\left|\gamma_{1}\right| \ll \omega$, the small-amplitude oscillations are sinusoidal with a slowly varying amplitude, $x=\operatorname{Re}\left[\alpha(t) e^{i \omega t}\right]$, where $\dot{\alpha}=\gamma_{1} \alpha-\gamma_{2}|\alpha|^{2} \alpha$ [4]. Thus, if $\gamma_{1}<0$, the system is fully damped and all oscillations die out, whereas for $\gamma_{1}>0$, they reach a stable amplitude with $|\alpha|=\sqrt{\gamma_{1} / \gamma_{2}}$. When subjected to a resonant drive $F \cos (\omega t+\phi)$, the equation of motion gains a forcing term,

$$
\dot{\alpha}=\gamma_{1} \alpha-\gamma_{2}|\alpha|^{2} \alpha+\Omega
$$

where $\Omega=-i e^{i \phi} F /(2 \omega)$. We choose $\phi=\pi / 2$ so that $\Omega$ is real and non-negative, without loss of generality. From Eq. (1), the steady-state response at the critical point $\gamma_{1}=0$ is purely nonlinear, $\alpha=\left(\Omega / \gamma_{2}\right)^{1 / 3}$. Hence, the susceptibility $\chi_{\mathrm{cl}} \equiv d \alpha / d \Omega$ is divergent at zero drive, $\chi_{\mathrm{cl}} \propto \Omega^{-2 / 3}$. Away from criticality, the response is of the form $\alpha=\tilde{\Omega}^{1 / 3} f\left(\tilde{\gamma}_{1} / \tilde{\Omega}^{2 / 3}\right)$, where $\tilde{\gamma}_{1} \equiv \gamma_{1} / \gamma_{2}$ and $\tilde{\Omega} \equiv \Omega / \gamma_{2}$ (details are in the Supplemental Material [28]). In particular, the limiting behavior is such that

$$
\alpha= \begin{cases}\sqrt{\gamma_{1} / \gamma_{2}}+\Omega /\left(2 \gamma_{1}\right) & \text { for } \tilde{\gamma}_{1} \gg \tilde{\Omega}^{2 / 3}, \\ \Omega /\left|\gamma_{1}\right| & \text { for } \tilde{\gamma}_{1} \ll-\tilde{\Omega}^{2 / 3}\end{cases}
$$

which are consistent with a diverging susceptibility, $\chi_{\mathrm{cl}} \propto 1 /\left|\gamma_{1}\right|$, at the critical point $\gamma_{1} \rightarrow 0$.

We study a quantum version of the vdP model, where a quantum harmonic oscillator is subjected to several 
dissipative processes, as in Refs. [21-27]. These are (i) oneparticle loss with rate $\gamma_{1}^{-}$, (ii) one-particle gain with rate $\gamma_{1}^{+}$, and (iii) two-particle loss with rate $\gamma_{2}$. As we will discuss later, such processes can be engineered in current experimental setups by coupling the oscillator to a suitable environment. Additionally, the oscillator is subject to a resonant drive of amplitude $\Omega$, given by the Hamiltonian $\hat{H}=\omega \hat{a}^{\dagger} \hat{a}+\Omega\left(i e^{i \omega t} \hat{a}^{\dagger}+\right.$ H.c. $)$. Here, $\hat{a}$ annihilates a particle in the oscillator mode, and $\hbar=1$. It is convenient to work in the rotating frame where the Hamiltonian reads $\hat{H}=i \Omega\left(\hat{a}^{\dagger}-\hat{a}\right)$. In general, the full dynamics of such a driven-dissipative system are governed by a master equation for the density matrix $\hat{\rho}$, obtained by tracing out the environment degrees of freedom [36]. However, for typical atomic or photonic setups, the environment relaxes to equilibrium on optical timescales, several orders of magnitude faster than the system dynamics [37]. Under such a routine Markov approximation $[38,39]$, the master equation reduces to the Lindblad form

$$
\dot{\hat{\rho}}=-i[\hat{H}, \hat{\rho}]+\gamma_{1}^{+} \mathcal{D}\left[\hat{a}^{\dagger}\right] \hat{\rho}+\gamma_{1}^{-} \mathcal{D}[\hat{a}] \hat{\rho}+\gamma_{2} \mathcal{D}\left[\hat{a}^{2}\right] \hat{\rho},
$$

where $\mathcal{D}[\hat{x}] \hat{\rho} \equiv \hat{x} \hat{\rho} \hat{x}^{\dagger}-\left\{\hat{x}^{\dagger} \hat{x}, \hat{\rho}\right\} / 2$ [40,41]. Using Eq. (3), or operator equations of motion [42], the expectation value $\langle\hat{a}\rangle$ can be shown to obey

$$
\langle\dot{\hat{a}}\rangle=\left[\left(\gamma_{1}^{+}-\gamma_{1}^{-}\right) / 2\right]\langle\hat{a}\rangle-\gamma_{2}\left\langle\hat{a}^{\dagger} \hat{a} \hat{a}\right\rangle+\Omega \text {. }
$$

Replacing $\hat{a}$ with a complex number $\alpha$ in Eq. (4) leads to the classical limit in Eq. (1) with $\gamma_{1}=\left(\gamma_{1}^{+}-\gamma_{1}^{-}\right) / 2$. Hence, the critical case corresponds to $\gamma_{1}^{+}=\gamma_{1}^{-}$. Note that, although Eq. (1) was derived in the small-amplitude limit, we will refer to Eq. (3) more generally as a quantum vdP oscillator, as in Refs. [21-27].

We find the response by numerically solving Eq. (3) for the steady-state density matrix, then computing $\langle\hat{a}\rangle=\operatorname{Tr}(\hat{a} \hat{\rho})$. It is most straightforward to use the Fock basis, $\hat{\rho}=\sum_{n n^{\prime}} \rho_{n n^{\prime}}|n\rangle\left\langle n^{\prime}\right|$, where $n=0,1,2, \ldots$ denotes the number of particles in the oscillator mode. The response is given by $\langle\hat{a}\rangle=\sum_{n} \sqrt{n} \rho_{n, n-1}$. Past studies have found closed-form expressions for $\rho_{n, n^{\prime}}$ in special cases [43-45].

First we consider the simplest critical case, $\gamma_{1}^{+}=\gamma_{1}^{-}=0$. Figure 1 shows the susceptibility $\chi \equiv d\langle\hat{a}\rangle / d \Omega$ as a function of drive. Note that it coincides with the classical result for $\langle\hat{a}\rangle \gtrsim 1$. However, at weaker drives, the classical divergence is cut off and $\chi$ saturates, producing a linear response. We can understand this low-energy cutoff as follows. At $\Omega=0$, both $|0\rangle$ and $|1\rangle$ are steady states, as there is only two-particle decay. A nonzero drive couples these neighboring Fock states, yielding $\rho_{10}, \rho_{21} \sim \Omega$ and $\rho_{22} \sim \Omega^{2}$. Hence, to linear order, one can restrict the dynamics to the lowest three levels, which gives $\langle\hat{a}\rangle=$ $2 \Omega / \gamma_{2}$ (see the Supplemental Material [28] for a

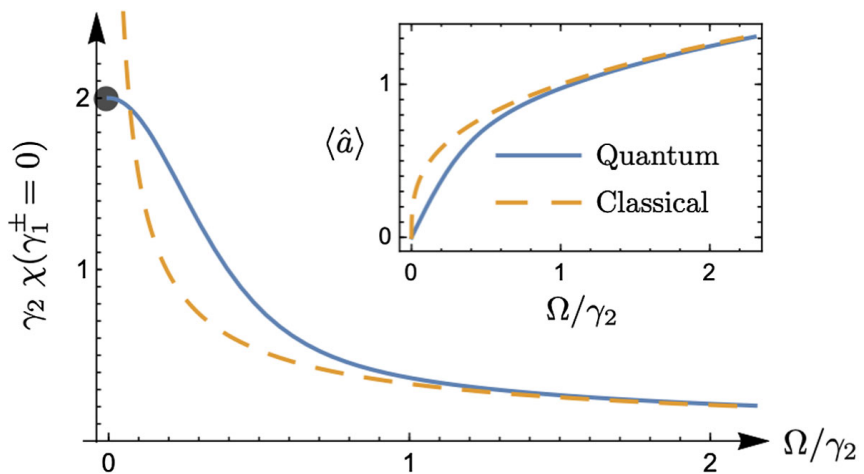

FIG. 1. Susceptibility $\chi$ and response $\langle\hat{a}\rangle$ of a resonantly driven van der Pol oscillator at the critical point $\gamma_{1}^{ \pm}=0$. Here, $\Omega$ is the drive amplitude, and $\gamma_{1}^{+}, \gamma_{1}^{-}$, and $\gamma_{2}$ are the rates of one-particle gain, one-particle loss, and two-particle loss, respectively. The classical and quantum responses match down to $\langle\hat{a}\rangle \sim 1$, below which quantum fluctuations cut off the classical divergence.

derivation). Stronger drives inject more particles, inducing a crossover to the classical limit.

We now consider departures from $\gamma_{1}^{ \pm}=0$ by allowing either $\gamma_{1}^{+}$or $\gamma_{1}^{-}$to be nonzero, spanning the transition from the quiescent $\left(\gamma_{1}^{+}=0, \gamma_{1}^{-}>0\right)$ to the limit-cycle regime $\left(\gamma_{1}^{+}>0, \gamma_{1}^{-}=0\right)$. In Fig. 2 , we plot the zero-drive susceptibility across the transition, which, remarkably, shows a divergence as $1 / \gamma_{1}^{ \pm}$at small damping or antidamping. This divergence can be understood from a competition of energy scales. For $\gamma_{1}^{+}=0$ and $\gamma_{1}^{-}>0$, the system is fully damped and the undriven steady state is $|0\rangle$. For weak drives $\Omega<\gamma_{1}^{-}$, the occupation remains small, so the two-particle decay is irrelevant. Thus, we can discard the nonlinear term in Eq. (4), which then reduces to a driven

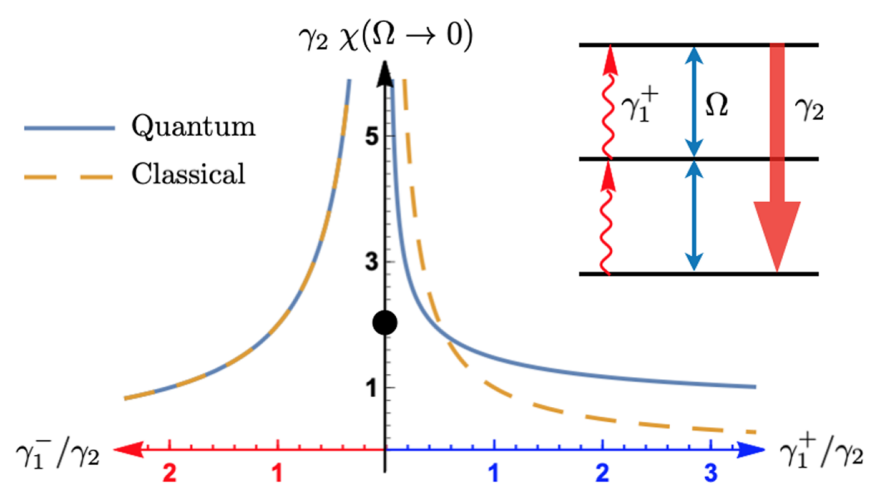

FIG. 2. Zero-drive susceptibility as a function of damping $\gamma_{1}^{-}$ (with $\gamma_{1}^{+}=0$, red axis) and antidamping $\gamma_{1}^{+}$(with $\gamma_{1}^{-}=0$, blue axis). Surprisingly, both classical and quantum susceptibilities diverge as $1 / \gamma_{1}^{ \pm}$, although at $\gamma_{1}^{ \pm}=0$, the quantum susceptibility is finite (black circle, also see Fig. 1). (Inset) Transitions among the lowest three oscillator levels for $\gamma_{1}^{+}, \Omega \ll \gamma_{2}$ and $\gamma_{1}^{-}=0$. Since any two-particle excitation decays very rapidly, the dynamics are confined to this low-energy subspace. 
damped oscillator with steady state $\langle\hat{a}\rangle=2 \Omega / \gamma_{1}^{-}$, matching the classical result. The response for $\gamma_{1}^{+}>0$ and $\gamma_{1}^{-}=0$ is more involved, as the oscillator can have large occupations even at zero drive. In general, the linear response is given by Liouvillian perturbation theory [46-48]. However, for $\gamma_{1}^{+} \ll \gamma_{2}$, the dynamics are confined to the lowest three levels, as shown in the Fig. 2 inset, which yields $\langle\hat{a}\rangle=$ $2 \Omega /\left(9 \gamma_{1}^{+}\right)$(for a derivation, see the Supplemental Material [28]).

At first sight, the results in Figs. 1 and 2 might appear to be contradictory: at $\gamma_{1}^{+}=\gamma_{1}^{-}=0$, the zero-drive susceptibility is finite (the black circle in Fig. 1); however, Fig. 2 shows that it diverges as the critical point is approached from either side. The resolution is that the susceptibility $\chi\left(\Omega \rightarrow 0, \gamma_{1}^{ \pm} \rightarrow 0\right)$ depends on the order of the two limits. This is better understood by plotting the full response as a function of drive for small damping or antidamping, as shown in Fig. 3. The response is strongly nonlinear for $\Omega \gtrsim \gamma_{1}^{ \pm}$. Thus, although the linear susceptibility diverges, the linear region itself shrinks to zero as $\gamma_{1}^{ \pm} \rightarrow 0$.

The full response exhibits four different regimes: (i) a linear response for $\Omega \lesssim \gamma_{1}^{ \pm}$, (ii) negative susceptibility for $\gamma_{1}^{ \pm} \lesssim \Omega \lesssim\left(\gamma_{1}^{ \pm} \gamma_{2}\right)^{1 / 2}$, (iii) an extended quantum response for $\left(\gamma_{1}^{ \pm} \gamma_{2}\right)^{1 / 2} \lesssim \Omega \lesssim \gamma_{2}$, and (iv) classical response for $\Omega \gtrsim \gamma_{2}$. The first three regions are well reproduced by a three-level approximation, which yields, to lowest order in $\gamma_{1}^{ \pm} / \gamma_{2}$ and $\Omega / \gamma_{2}$ (see the Supplemental Material [28]),

$$
\langle\hat{a}\rangle \approx \frac{2 \Omega}{\gamma_{2}}\left(\frac{\left(\gamma_{1}^{+}+\gamma_{1}^{-}\right) \gamma_{2}+8 \Omega^{2}}{\left(3 \gamma_{1}^{+}+\gamma_{1}^{-}\right)^{2}+8 \Omega^{2}}\right) .
$$

Hence, in the linear region, $\chi \approx 2\left(\gamma_{1}^{+}+\gamma_{1}^{-}\right) /\left(3 \gamma_{1}^{+}+\gamma_{1}^{-}\right)^{2}$, which diverges for $\gamma_{ \pm} \rightarrow 0$, whereas for $\Omega \sim\left(\gamma_{1}^{ \pm} \gamma_{2}\right)^{1 / 2}$, $\langle\hat{a}\rangle \approx 2 \Omega / \gamma_{2}+\left(\gamma_{1}^{+}+\gamma_{1}^{-}\right) /(4 \Omega)$, which exhibits a local minimum. This nonmonotonic response is also evident in the Wigner functions shown in the lower panel of Fig. 3, which describe a quasiprobability distribution in phase space [41]. The Wigner function is widely used as an integral representation of the density matrix (the Supplemental Material [28] contains a brief review).

Physically, the separate regions originate from an interplay between drive and dissipation within a few-level manifold. Consider the case $\gamma_{1}^{+}=0$ and $0<\gamma_{1}^{-} \ll \gamma_{2}$. A weak drive couples the steady state $|0\rangle$ to $|1\rangle$, producing a coherence $\rho_{10} \sim \Omega / \gamma_{1}^{-}$. However, $\rho_{11}$ also grows with $\Omega$ and saturates at $1 / 2$. This saturation acts as negative feedback for $\rho_{10}$, as seen from the equation of motion $\dot{\rho}_{11}=2 \Omega \rho_{10}-\gamma_{1}^{-} \rho_{11}$. Thus, for $\rho_{11} \approx 1 / 2, \rho_{10}$ falls off as $\gamma_{1}^{-} /(4 \Omega)$, giving rise to negative susceptibility. At larger drives, higher-energy modes become accessible and coherences can grow again. Then the response is similar to the case $\gamma_{1}^{ \pm}=0$, with a purely quantum regime for $\langle\hat{a}\rangle \lesssim 1$ and a classical regime for $\langle\hat{a}\rangle \gtrsim 1$ (cf. Fig. 1). A similar variation with drive is found for $0<\gamma_{1}^{+} \ll \gamma_{2}$.

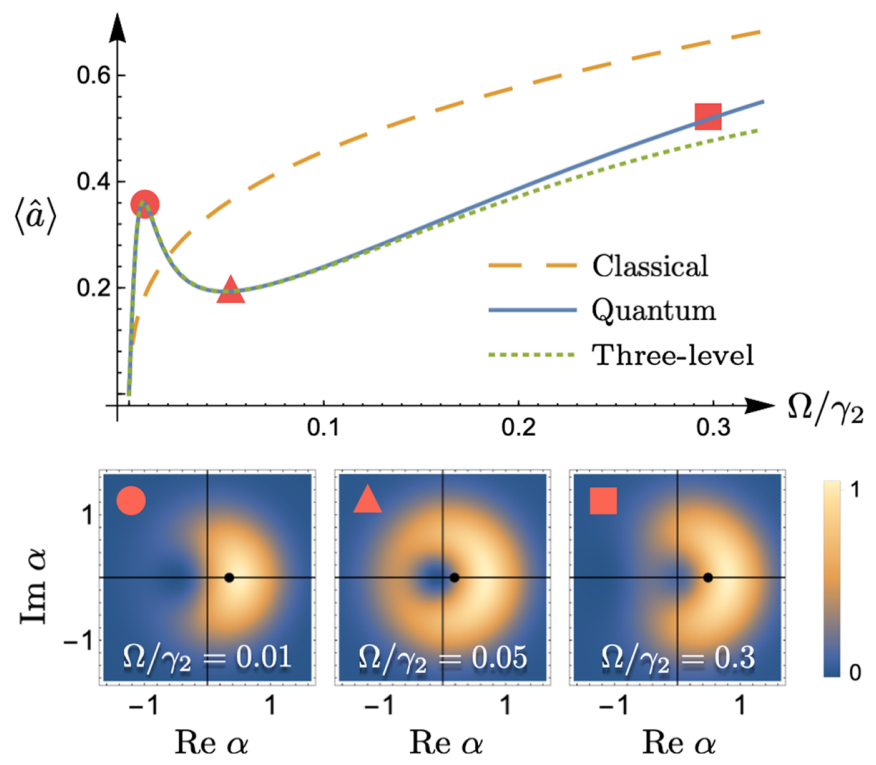

FIG. 3. (Top panel) Response $\langle\hat{a}\rangle$ as a function of drive $\Omega$ for $\gamma_{1}^{+}=0$ and $\gamma_{1}^{-} / \gamma_{2}=0.02$. The quantum response is linear for $\Omega \lesssim \gamma_{1}^{-}$and shows negative susceptibility for $\gamma_{1}^{-} \lesssim \Omega \lesssim\left(\gamma_{1}^{-} \gamma_{2}\right)^{1 / 2}$. For $\Omega \gtrsim \gamma_{2}$, it agrees with the classical response, as in Fig. 1. (Bottom panels) Rescaled Wigner functions $W(\alpha)$ describing a quasiprobability distribution in phase space [28]. The response $\langle\hat{a}\rangle$ is given by the center of mass of $W(\alpha)$, shown by black dots.

Note that the concomitant divergent and negative susceptibilities are genuine quantum features which result from a very general set of conditions involving a competition between coherent and incoherent energy scales. Thus, we expect them to show up generically in driven-dissipative systems. For the vdP oscillator, we find that they are robust to anharmonicity and detuning.

We have shown the quantum system exhibits a diverging linear susceptibility close to a critical point (Fig. 2), qualitatively similar to the classical system. Can this be advantageous in quantum sensing applications? More precisely, can one enhance the sensitivity of a damped quantum oscillator by introducing an incoherent pump? For such a passive oscillator, the equation of motion simply reads $\dot{\hat{a}}=\Omega-\left(\gamma_{1}^{-} / 2\right) \hat{a}$, so the steady state is $\langle\hat{a}\rangle=2 \Omega / \gamma_{1}^{-}$ and the passive susceptibility is $\chi_{p}=2 / \gamma_{1}^{-}$. To discuss whether pumping enhances sensitivity, we define the sensitivity gain at weak drives, $G_{0} \equiv \chi /\left.\chi_{p}\right|_{\Omega \rightarrow 0}$.

One regime where there is a gain, $G_{0}>1$, is the classical limit. Then the response is only a function of $\gamma_{1}^{+}-\gamma_{1}^{-}$, and one can simply set $\gamma_{1}^{+}=\gamma_{1}^{-}$to make the system infinitely sensitive to weak signals for any damping. Quantum mechanically, however, the response also depends on the average $\Gamma_{1} \equiv\left(\gamma_{1}^{+}+\gamma_{1}^{-}\right) / 2$. Figure 4 shows how the classical limit emerges with increasing $\Gamma_{1}$ for the critical case, $\gamma_{1}^{+}=\gamma_{1}^{-}$. We see that $G_{0}$ scales as $\sqrt{\Gamma_{1} / \gamma_{2}}$, and one can have $\chi>\chi_{p}$ provided $\gamma_{1}^{-} \gtrsim 3 \gamma_{2}$, which holds for typical experimental systems [49]. The relatively slow growth of 


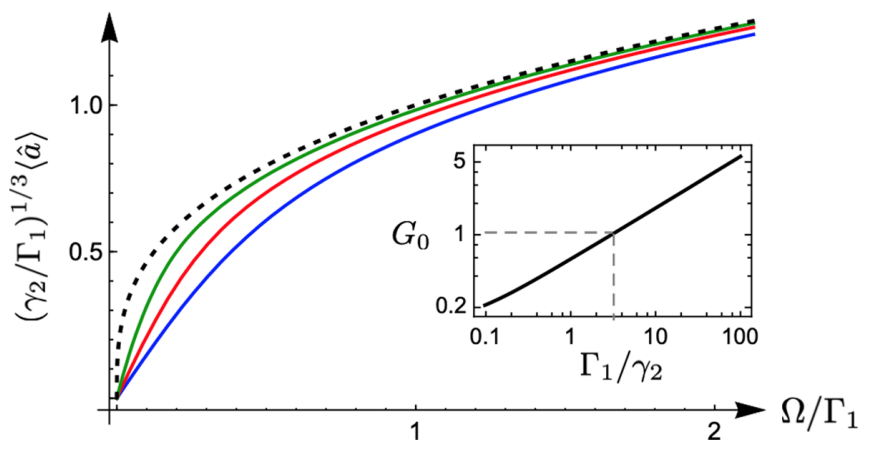

FIG. 4. Response for the critical condition $\gamma_{1}^{+}=\gamma_{1}^{-}=\Gamma_{1}$ with $\Gamma_{1} / \gamma_{2}=5$ (blue line), 50 (red line), and 1000 (green line), showing the approach to classical limit (dotted black line). (Inset) Sensitivity gain over a passive oscillator, $G_{0} \sim \sqrt{\Gamma_{1} / \gamma_{2}}$.

$G_{0}$ with $\Gamma_{1}$ stems from large number fluctuations at criticality (which also limits the sensing efficiency, see below). Since $\gamma_{1}^{+}=\gamma_{1}^{-}$, the undriven system would reach an infinite-temperature state in the limit $\gamma_{2} \rightarrow 0$. A nonzero $\gamma_{2}$ results in a Gaussian number distribution of width $\sqrt{\Gamma_{1} / \gamma_{2}}$ [44], leading to the linear susceptibility $\chi \approx 2 / \sqrt{\pi \Gamma_{1} \gamma_{2}}$ (see the Supplemental Material [28]).

The sensitivity can be enhanced further by operating deep in the limit-cycle phase, $\gamma_{1}^{+}-\gamma_{1}^{-} \gg \gamma_{2}$, as in Fig. 5. Here, a vanishingly small drive breaks the phase-rotation symmetry, yielding a nonzero classical response. For the quantum oscillator, the coherence $\langle\hat{a}\rangle$ builds up at a finite rate (see the Supplemental Material for a derivation [28])

$$
\chi \approx \frac{2}{3 \gamma_{2}}\left(1-\frac{2 \gamma_{1}^{-}}{3 \gamma_{1}^{+}}\right) .
$$

Hence, the susceptibility is limited only by $\gamma_{2}$, and one can obtain a large enhancement. In particular, for $\gamma_{1}^{+} \gg \gamma_{1}^{-}$, $G_{0} \approx \gamma_{1}^{-} /\left(3 \gamma_{2}\right) \gg 1$. In other words, a sufficiently strong incoherent pump can negate the linear damping and yield a response bounded only by two-body loss. This heightened sensitivity persists until the oscillator switches over to the classical limit at $\Omega \gtrsim\left(\gamma_{1}^{+} \gamma_{2}\right)^{1 / 2}$, as shown in Fig. 5. Such a

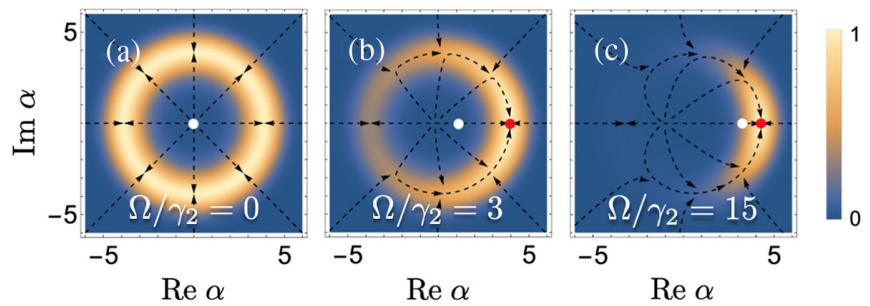

FIG. 5. (a)-(c) Classical trajectories and rescaled Wigner functions at increasing drive for $\gamma_{1}^{+} / \gamma_{2}=50$ and $\gamma_{1}^{-} / \gamma_{2}=20$. The quantum and classical responses are shown by white and red dots, respectively. As the quantum system crosses over from a symmetry-broken limit cycle to the classical steady state, its susceptibility is limited only by two-body loss. behavior is reminiscent of sensing protocols based on switching in optically bistable systems [50].

Apart from the "signal" $\langle\hat{a}\rangle$, the efficiency of sensors is limited by the signal-to-noise ratio (SNR) [10,51]. Estimating the noise from the spread of the Wigner function, we find one can have $G_{0}>1$ and SNR $\gtrsim 1$ in the quantum domain, $\langle\hat{a}\rangle \sim 1$ (see the Supplemental Material [28]). Further, one can measure weak signals in fewer shots in the limit-cycle regime than in the classical limit of the critical oscillator. This is because particle-number fluctuations diverge in this limit, causing the SNR to vanish.

The quantum vdP model can be probed in several experimental setups. In particular, as proposed in Refs. [21,22], one can engineer the dissipation via sideband transitions which either add or remove energy quanta. For instance, one can laser excite a harmonically trapped ion to its blue or red motional sidebands to implement one-phonon gain or two-phonon loss, as illustrated in Fig. 6. In such a setup, the environment relaxation time is of order $\Delta E^{-1}$, where $\Delta E$ is the level spacing between $|g\rangle$ and $|e\rangle$, which dominates all other energy scales [37]. Hence, the Markov approximation holds. Additionally, one must have resolved sidebands and suppress off-resonant excitations. As shown in Ref. [21], one can satisfy these constraints for several tens of low-energy modes. The response to a resonant drive can be measured with well-established techniques, including tomography [52] and more direct mapping of the Wigner function [53-57].

Similar techniques can be used for phonon modes of an optomechanical membrane [22] or photon modes in microwave resonators [58]. One can also realize an incoherent pump through spontaneous emission from a set of inverted qubits $[59,60]$. Strong two-body loss can result from Josephson junctions in superconducting circuits [49] or polariton blockade in an optical cavity [61].

Finally, note that Eq. (3) is not the only quantum model that reproduces the classical limit of the vdP oscillator. In particular, one can harness energy-dependent onebody loss instead of two-body loss, as in Refs. [62-65]. Numerics show that the key features are unaffected.

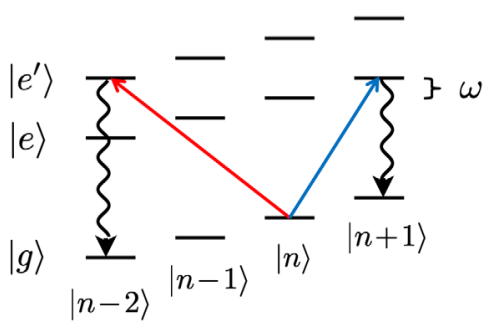

FIG. 6. An experimental scheme for realizing a quantum vdP oscillator with a trapped ion, adapted from Ref. [21]. Motional modes $|n\rangle$ act as the energy levels of the oscillator, while $|g\rangle,|e\rangle$, and $\left|e^{\prime}\right\rangle$ represent internal states. Sideband transitions, followed by spontaneous decay, effectively inject or remove energy quanta (phonons), realizing gain and loss processes. 
There may also exist alternative descriptions of the $\mathrm{vdP}$ oscillator in terms of nonstandard Hamiltonians with possible quantization [66-68].

In summary, we characterize in this Letter the response of an open quantum system across a dynamical critical point by modeling a prototypical self-sustained oscillator. Like its classical counterpart, we find an increased sensitivity to external drives, which is ideal for sensing applications. Genuine quantum features arise at very weak drives, including a power-law diverging linear susceptibility and a concomitant negative susceptibility. To further explore the prospects of sensing, we compare the response to that of a passive system, where the sensitivity is limited by one-body loss. We show that this linear damping is negated by a strong incoherent pump, yielding a response bounded only by weak nonlinear effects, e.g., two-body decay. Our findings are largely generic and can be probed in present-day experimental platforms, contributing to a broader understanding of dynamical criticality [9] and quantum-to-classical crossover $[69,70]$.

We thank Andreas Nunnenkamp and Chris Parmee for the helpful discussions. This work was supported by EPSRC Grant No. EP/P009565/1 and by a Simons Investigator Award.

*sd843@cam.ac.uk †nrc25@cam.ac.uk

[1] M. Kardar, Statistical Physics of Fields (Cambridge University Press, Cambridge, England, 2007).

[2] S. H. Strogatz, Nonlinear Dynamics and Chaos (Westview Press, Cambridge, MA, 1994).

[3] J. E. Marsden and M. McCracken, The Hopf Bifurcation and its Applications (Springer-Verlag, New York, 1976).

[4] B. van der Pol, LXXXVIII. On "relaxation-oscillations," Philos. Mag. 2, 978 (1926).

[5] T. A. J. Duke and F. Jülicher, Critical oscillators as active elements in hearing, in Active Processes and Otoacoustic Emissions in Hearing, edited by G. A. Manley, R. R. Fay, and A. N. Popper (Springer, New York, 2008), p. 63.

[6] V. M. Eguíluz, M. Ospeck, Y. Choe, A. J. Hudspeth, and M. O. Magnasco, Essential Nonlinearities in Hearing, Phys. Rev. Lett. 84, 5232 (2000).

[7] T. Mora and W. Bialek, Are biological systems poised at criticality?, J. Stat. Phys. 144, 268 (2011).

[8] M. A. Munõz, Colloquium: Criticality and dynamical scaling in living systems, Rev. Mod. Phys. 90, 031001 (2018)

[9] A. Roli, M. Villani, A. Filisetti, and R. Serra, Dynamical criticality: Overview and open questions, J. Syst. Sci. Complexity 31, 647 (2018).

[10] C. L. Degen, F. Reinhard, and P. Cappellaro, Quantum sensing, Rev. Mod. Phys. 89, 035002 (2017).

[11] D. Braun, G. Adesso, F. Benatti, R. Floreanini, U. Marzolino, M. W. Mitchell, and S. Pirandola, Quantum-enhanced measurements without entanglement, Rev. Mod. Phys. 90, 035006 (2018).
[12] L. M. Sieberer, M. Buchhold, and S. Diehl, Keldysh field theory for driven open quantum systems, Rep. Prog. Phys. 79, 096001 (2016).

[13] I. Rotter and J. P. Bird, A review of progress in the physics of open quantum systems: Theory and experiment, Rep. Prog. Phys. 78, 114001 (2015).

[14] M. Müller, S. Diehl, G. Pupillo, and P. Zoller, Engineered open systems and quantum simulations with atoms and ions, Adv. At. Mol. Opt. Phys. 61, 1 (2012).

[15] A. A. Houck, H. E. Türeci, and J. Koch, On-chip quantum simulation with superconducting circuits, Nat. Phys. 8, 292 (2012).

[16] M. Aspelmeyer, T. J. Kippenberg, and F. Marquardt, Cavity optomechanics, Rev. Mod. Phys. 86, 1391 (2014).

[17] C. Noh and D. G. Angelakis, Quantum simulations and many-body physics with light, Rep. Prog. Phys. 80, 016401 (2017).

[18] I. M. Georgescu, S. Ashhab, and F. Nori, Quantum simulation, Rev. Mod. Phys. 86, 153 (2014).

[19] K. Vahala, M. Herrmann, S. Knünz, V. Batteiger, G. Saathoff, T. W. Hänsch, and Th. Udem, A phonon laser, Nat. Phys. 5, 682 (2009).

[20] M. Sargent III, M. O. Scully, and W. E. Lamb, Jr, Laser Physics (Addison-Wesley, Reading, MA, 1974).

[21] T. E. Lee and H. R. Sadeghpour, Quantum Synchronization of Quantum van der Pol Oscillators with Trapped Ions, Phys. Rev. Lett. 111, 234101 (2013).

[22] S. Walter, A. Nunnenkamp, and C. Bruder, Quantum Synchronization of a Driven Self-Sustained Oscillator, Phys. Rev. Lett. 112, 094102 (2014).

[23] N. Lörch, E. Amitai, A. Nunnenkamp, and C. Bruder, Genuine Quantum Signatures in Synchronization of Anharmonic Self-Oscillators, Phys. Rev. Lett. 117, 073601 (2016).

[24] T. E. Lee, C.-K. Chan, and S. Wang, Entanglement tongue and quantum synchronization of disordered oscillators, Phys. Rev. E 89, 022913 (2014).

[25] S. Walter, A. Nunnenkamp, and C. Bruder, Quantum synchronization of two van der Pol oscillators, Ann. Phys. (Berlin) 527, 131 (2015).

[26] K. Ishibashi and R. Kanamoto, Oscillation collapse in coupled quantum van der Pol oscillators, Phys. Rev. E 96, 052210 (2017).

[27] O. Scarlatella, A. A. Clerk, and M. Schiro, Spectral functions and negative density of states of a driven-dissipative nonlinear quantum resonator, New J. Phys. 21, 043040 (2019).

[28] See Supplemental Material at http://link.aps.org/ supplemental/10.1103/PhysRevLett.123.250401, which includes Refs. [29-35], for exact classical solution, analytic results for the quantum response at weak drives, mapping between density matrix and the Wigner function, and signalto-noise ratio estimates.

[29] E. Wigner, On the quantum correction for thermodynamic equilibrium, Phys. Rev. 40, 749 (1932).

[30] K.E. Cahill and R. J. Glauber, Density operators and quasiprobability distributions, Phys. Rev. 177, 1882 (1969).

[31] K. E. Cahill and R. J. Glauber, Ordered expansions in boson amplitude operators, Phys. Rev. 177, 1857 (1969). 
[32] A. I. Lvovsky, H. Hansen, T. Aichele, O. Benson, J. Mlynek, and S. Schiller, Quantum State Reconstruction of the SinglePhoton Fock State, Phys. Rev. Lett. 87, 050402 (2001).

[33] M. Hofheinz et al., Synthesizing arbitrary quantum states in a superconducting resonator, Nature (London) 459, 546 (2009).

[34] Y. Chu, P. Kharel, T. Yoon, L. Frunzio, P. T. Rakich, and R. J. Schoelkopf, Creation and control of multi-phonon Fock states in a bulk acoustic-wave resonator, Nature (London) 563, 666 (2018).

[35] D. T. Smithey, M. Beck, M. G. Raymer, and A. Faridani, Measurement of the Wigner Distribution and the Density Matrix of a Light Mode Using Optical Homodyne Tomography: Application to Squeezed States and the Vacuum, Phys. Rev. Lett. 70, 1244 (1993).

[36] R. R. Puri and S. V. Lawande, On exact master equation for an open system, Phys. Lett. 62A, 143 (1977).

[37] A. J. Daley, Quantum trajectories and open many-body quantum systems, Adv. Phys. 63, 77 (2014).

[38] Á. Rivas, A. D. K. Plato, S. F. Huelga, and M. B. Plenio, Markovian master equations: A critical study, New J. Phys. 12, 113032 (2010).

[39] Á. Rivas, S. F. Huelga, and M. B. Plenio, Quantum nonMarkovianity: Characterization, quantification and detection, Rep. Prog. Phys. 77, 094001 (2014).

[40] H.-P. Breuer and F. Petruccione, The Theory of Open Quantum Systems (Oxford University Press, Oxford, 2002).

[41] C. Gardiner and P. Zoller, Quantum Noise (Springer, New York, 2004).

[42] S. Sachdev, Atom in a damped cavity, Phys. Rev. A 29, 2627 (1984).

[43] H. D. Simaan and R. Loudon, Quantum statistics of singlebeam two-photon absorption, J. Phys. A 8, 539 (1975).

[44] V. V. Dodonov and S. S. Mizrahi, Exact stationary photon distributions due to competition between one- and twophoton absorption and emission, J. Phys. A 30, 5657 (1997).

[45] K. V. Kheruntsyan, Wigner function for a driven anharmonic oscillator, J. Opt. B 1, 225 (1999).

[46] A. C. Y. Li, F. Petruccione, and J. Koch, Perturbative approach to Markovian open quantum systems, Sci. Rep. 4, 4887 (2014).

[47] V. V. Albert, B. Bradlyn, M. Fraas, and L. Jiang, Geometry and Response of Lindbladians, Phys. Rev. X 6, 041031 (2016).

[48] M. Konopik and E. Lutz, Quantum response theory for nonequilibrium steady states, Phys. Rev. Research 1, 033156 (2019).

[49] Z. Leghtas et al., Confining the state of light to a quantum manifold by engineered two-photon loss, Science 347, 853 (2015).

[50] S. Aldana, C. Bruder, and A. Nunnenkamp, Detection of weak forces based on noise-activated switching in bistable optomechanical systems, Phys. Rev. A 90, 063810 (2014).

[51] H.-K. Lau and A. A. Clerk, Fundamental limits and nonreciprocal approaches in non-Hermitian quantum sensing, Nat. Commun. 9, 4320 (2018).

[52] A. I. Lvovsky and M. G. Raymer, Continuous-variable optical quantum-state tomography, Rev. Mod. Phys. 81, 299 (2009).
[53] K. Banaszek and K. Wódkiewicz, Direct Probing of Quantum Phase Space by Photon Counting, Phys. Rev. Lett. 76, 4344 (1996).

[54] K. Banaszek, C. Radzewicz, K. Wódkiewicz, and J. S. Krasiński, Direct measurement of the Wigner function by photon counting, Phys. Rev. A 60, 674 (1999).

[55] D. Leibfried, D. M. Meekhof, B. E. King, C. Monroe, W. M. Itano, and D. J. Wineland, Experimental Determination of the Motional Quantum State of a Trapped Atom, Phys. Rev. Lett. 77, 4281 (1996).

[56] L. G. Lutterbach and L. Davidovich, Method for Direct Measurement of the Wigner Function in Cavity QED and Ion Traps, Phys. Rev. Lett. 78, 2547 (1997).

[57] P. Bertet, A. Auffeves, P. Maioli, S. Osnaghi, T. Meunier, M. Brune, J. M. Raimond, and S. Haroche, Direct Measurement of the Wigner Function of a One-Photon Fock State in a Cavity, Phys. Rev. Lett. 89, 200402 (2002).

[58] J. R. Johansson, G. Johansson, and F. Nori, Optomechanicallike coupling between superconducting resonators, Phys. Rev. A 90, 053833 (2014).

[59] J. Lebreuilly, M. Wouters, and I. Carusotto, Towards strongly correlated photons in arrays of dissipative nonlinear cavities under a frequency-dependent incoherent pumping, C. R. Phys. 17, 836 (2016).

[60] R. Ma, C. Owens, A. Houck, D. I. Schuster, and J. Simon, Autonomous stabilizer for incompressible photon fluids and solids, Phys. Rev. A 95, 043811 (2017).

[61] N. Jia, N. Schine, A. Georgakopoulos, A. Ryou, L. W. Clark, A. Sommer, and J. Simon, A strongly interacting polaritonic quantum dot, Nat. Phys. 14, 550 (2018).

[62] N. Lörch, S. E. Nigg, A. Nunnenkamp, R. P. Tiwari, and C. Bruder, Quantum Synchronization Blockade: Energy Quantization Hinders Synchronization of Identical Oscillators, Phys. Rev. Lett. 118, 243602 (2017).

[63] S. E. Nigg, Observing quantum synchronization blockade in circuit quantum electrodynamics, Phys. Rev. A 97, 013811 (2018).

[64] J. J. Viennot, X. Ma, and K. W. Lehnert, Phonon-NumberSensitive Electromechanics, Phys. Rev. Lett. 121, 183601 (2018).

[65] S. Rips, M. Kiffner, I. Wilson-Rae, and M. J. Hartmann, Steady-state negative Wigner functions of nonlinear nanomechanical oscillators, New J. Phys. 14, 023042 (2012).

[66] V. K. Chandrasekar, M. Senthilvelan, and M. Lakshmanan, On the Lagrangian and Hamiltonian description of the damped linear harmonic oscillator, J. Math. Phys. (N.Y.) 48, 032701 (2007).

[67] C. M. Bender, M. Gianfreda, N. Hassanpour, and H. F. Jones, Comment on "On the Lagrangian and Hamiltonian description of the damped linear harmonic oscillator" [J. Math. Phys. 48, 032701 (2007)], J. Math. Phys. (N.Y.) 57, 084101 (2016).

[68] V. Chithiika Ruby, M. Senthilvelan, and M. Lakshmanan, Exact quantization of a PT-symmetric (reversible) Liénardtype nonlinear oscillator, J. Phys. A 45, 382002 (2012).

[69] L. Davidovich, From quantum to classical: Schrödinger cats, entanglement, and decoherence, Phys. Scr. 91, 063013 (2016).

[70] M. A. Schlosshauer, Quantum decoherence, Phys. Rep. 831, 1 (2019). 